\title{
Sequential Auctions in Uncertain Information Settings
}

\author{
Shaheen S. Fatima ${ }^{1} \quad$ Michael Wooldridge $^{1} \quad$ Nicholas R. Jennings $^{2}$ \\ ${ }^{1}$ Department of Computer Science \\ University of Liverpool, Liverpool L69 3BX, U.K. \\ \{shaheen, mjw\}ecsc.liv.ac.uk \\ ${ }^{2}$ School of Electronics and Computer Science \\ University of Southampton, Southampton SO17 1BJ, U.K. \\ nrjeecs.soton.ac.uk
}

\begin{abstract}
This paper analyzes sequential auctions for private value objects using second-price sealed-bid rules. Now, the equilibrium bids for such auctions depend on the information uncertainty of the bidders. Specifically, there are three key auction parameters that the bidders could be uncertain about: the valuations of the objects for sale, the number of objects for sale, and the number of participating bidders. We analyse the bidding behaviour for each of these three sources of uncertainty. For each setting, we first find the equilibrium bidding strategies for the individual auctions that comprise a series. Then we analyze the effect of these uncertainties on the computational and economic properties of the equilibrium solution. The former analysis is essential if we want to use software agents to bid on our behalf. The latter is essential because both the auctioneer and the bidders want to know how these uncertainties affect their profits. Thus we compare the outcomes for these settings from the perspective of the bidders (i.e., in terms of their profits), from the perspective of the auctioneer (i.e., in terms of his revenue), and from a global perspective (i.e., in terms of auction efficiency).
\end{abstract}

\section{Introduction}

Auctions are now being widely studied as a means of buying/selling resources in multiagent systems. This is because auctions are not only simple but can also have desirable economic properties, probably the most important of which are their ability to generate high revenues to the seller and also allocate resources effi ciently $[13,2,15]$. Now, in many cases the number of objects to be auctioned is more than one. For such cases, there are two primary types of auctions: combinatorial [12] and sequential $[4,1]$. The former are typically used when the objects for sale are all available at the same time, while the latter are used when the objects become available at different points in time. Our paper focuses on sequential auctions. For these, it has been shown that although there is only one object being auctioned at a time, the bidding behaviour for any individual auction strongly depends on the auctions that are yet to be conducted $[4,1]$.

Given this, a key problem in the area of sequential auctions is to study the strategic behaviour of bidders in each individual auction. To date, considerable research effort has been devoted to this problem, but most of this work has focussed on scenarios where the bidders are uncertain about the other bidders' valuations for the objects. However, 
this is but one source of uncertainty, and there are other auction parameters that the bidders could equally well be uncertain ${ }^{1}$ about. Moreover, these different cases lead to different bidding behaviour and consequently result in different outcomes.

Against this background, our objective is to analyse the bidding behaviour for a range of uncertain information settings. Specifi cally, we analyse four incomplete information settings where the bidders are uncertain about:

$S_{1}$. The other bidders' valuations for the objects.

$S_{2}$. The other bidders' valuations and the number of objects for sale.

$S_{3}$. The other bidders' valuations and the number of participating bidders.

$S_{4}$. The other bidders' valuations, the number of objects for sale, and the number of participating bidders.

For each of these four settings, we fi rst fi nd the equilibrium bidding strategies for the individual auctions that comprise a series. Then we analyze the effect of these uncertainties on the computational and economic properties of the equilibrium solution. The former analysis is essential if we want to use software agents to bid on our behalf. The latter is essential because both the auctioneer and the bidders want to know how these uncertainties affect their profi ts. Thus we compare the outcomes for these settings from the perspective of the bidders (i.e., in terms of their profi ts), from the perspective of the auctioneer (i.e., in terms of his revenue), and from a global perspective (i.e., in terms of auction effi ciency).

Our study shows that, provided the bidders pre-compute certain functions (which we defi ne in terms of their common knowledge about the auction parameters) before the auctions begin, the equilibrium bids can be computed in constant time for all the four scenarios. We also show that between the four scenarios, $S_{1}$ yields maximum profi to the bidders, and $S_{4}$ yields maximum revenue. However, the effi ciency remains the same in all the scenarios.

The remainder of the paper is organized as follows. Section 2 describes the auction setting. Sections 3 to 6 determine the equilibrium bids for the four information settings. Section 7 discusses related literature, and Section 8 concludes.

\section{The Auction Setting}

This model is a generalisation of [1], which studies sequential auctions for two private value objects in the above defi ned setting $S_{1}$. Here, we generalise this model to $m>2$ objects and also analyse it in three different information settings. There are $m$ private value objects for sale. Each object is sold in a separate auction using the second-price sealed-bid rules and the auctions are held sequentially. There are $n$ risk neutral bidders. The valuations for the $m$ objects are independently and identically distributed across the bidders. Let $V_{j}: R_{+} \rightarrow[0,1](1 \leq j \leq m)$ denote the probability distribution function for the valuation for object $j$.

\footnotetext{
${ }^{1}$ These other uncertainties have been studied, but mostly for single object auctions - as in [8]. Our objective is to analyze multi-object sequential auctions with different sources of uncertainty.
} 
The sequential auctions are conducted as follows. The fi rst object is sold in a secondprice sealed bid auction. There are $n$ bidders for this auction. The winning bid for the fi rst auction is announced at the end of the auction. Each bidder needs a single object. Thus the winning bidder for an auction does not participate in any of the subsequent auctions. All the losing bidders for an auction go to the next auction. This process repeats for each of the $m$ objects. In other words, the bidders continue to bid in the auctions only until they win an object. Thus, if there are $n$ bidders in the fi rst auction, then there are $n-1$ bidders for the second, $n-2$ for the third, and so on. In general, there are $n-j+1$ bidders for auction $j(1 \leq j \leq m)$.

Given this context, we analyse the four different incomplete information settings $S_{1}$, $S_{2}, S_{3}$, and $S_{4}$ defi ned in Section 1. Note that a bidder's uncertainty about the others' valuations is common to all the settings. The auctions are conducted as follows. To begin, the p.d.fs $V_{j}(1 \leq j \leq m)$ are common knowledge to all the bidders. However, each bidder draws his private value signal for auction $j$ after the end of auction $j-1$. Thus, although the p.d.fs for all the objects are initially known to all the bidders, a bidder comes to know his valuation for auction $j$ only just before the auction begins.

In more detail, the auctions are held as follows: i) All the bidders draw their private value signals for auction 1 from the p.d.f. $V_{1}$. ii) Auction 1 is held using the second-price sealed-bid rules; at the end of the auction, the object is allocated to the winning bidder. iii) The winning bidder for auction 1 leaves (because each bidder has unit demand) and the remaining bidders go to the next auction. iv) For the next auction, the bidders draw their private value signals (signals for auction $1 \leq j \leq m$ are drawn from the p.d.f. $V_{j}$ ). v) Steps ii to iv are repeated for each of the remaining objects.

Note that the private values for our model are not correlated across the $m$ objects. Such correlations would occur if the value for object $j=2, \ldots, m$ can be determined on the basis of the value for object $k<j$. However, our present work focuses on $m$ dissimilar objects, where such a direct relation between the objects may not exist. Hence, for our analysis, the different objects have different distribution functions.

\section{Equilibrium bids for scenario $S_{1}$}

In this setting, the p.d.fs for the valuations of the objects (i.e., $V_{j}$ for $1 \leq j \leq m$ ), the number of objects $(m)$, the number of bidders for the fi rst auction $(n)$, and the auction agenda are common knowledge to the bidders.

Since there is more than one auction, a bidder's bid for an auction depends not only on that auction but also on the profi $t$ he expects to get from the future auctions. This profi $t$ depends on the number of bidders that participate in the future auctions. Given this, we fi rst determine this profi $\mathrm{t}$ and then fi nd the equilibrium bids.

If the number of bidders for the first auction is $n$, then let $\beta_{1}(y, j, m, n)$ denote a bidder's ex-ante probability of winning the $y$ th (for $j \leq y \leq m$ ) auction in the series from the $j$ th to the $m$ th one before the $j$ th auction begins. For instance, consider $\beta_{1}(1,1, m, n)$, which is the probability of winning the fi rst auction in the series of auctions from the fi rst to the $m$ th one. Since $\beta_{1}(1,1, m, n)$ is the ex-ante probability (i.e., before the bidders draw their values for the fi rst auction), each bidder has equal 
chances of winning the fi rst auction, i.e., $\beta_{1}(1,1, m, n)=1 / n$. Recall from Section 2 that if a bidder wins the fi rst auction, he does not participate in the remaining ones.

Now consider the ex-ante probability $\beta_{1}(2,1, m, n)$, which is the probability that a bidder wins the second auction in the series of auctions from the first to the $m$ th one where $\beta_{1}(2,1, m, n)=(1-1 / n)(1 /(n-1))=1 / n$. This is because a bidder can win the second auction if he loses the fi rst one - this has probability $(1-1 / n)$. The probability of winning the second auction is $1 /(n-1)$. If he wins the second auction then he does not participate in the remaining auctions. In the same way, for $1 \leq y \leq m$, we get $\beta_{1}(y, 1, m, n)$ as:

$$
\beta_{1}(y, 1, m, n)=\frac{1}{n-y+1} \prod_{k=1}^{y-1}\left(1-\frac{1}{n-k+1}\right)=\frac{1}{n-y+1} \prod_{k=1}^{y-1} \frac{n-k}{n-k+1}=\frac{1}{n} .
$$

In general, for $j \leq y \leq m, \beta_{1}(j, y, m, n)$ is given by:

$\beta_{1}(y, j, m, n)=\frac{1}{n-y+1} \prod_{k=j}^{y-1}\left(1-\frac{1}{n-k+1}\right)=\frac{1}{n-y+1} \prod_{k=j}^{y-1} \frac{n-k}{n-k+1}=\frac{1}{n-j+1}$.

Note that $\beta_{1}(y, j, m, n)$ does not depend on $y$. Intuitively, before the beginning of the $j$ th auction, all bidders are symmetric with respect to winning the $y$ th auction, and there are $n-j+1$ bidders left at that point. Hence, each bidder's probability of winning the $y$ th auction is $1 /(n-j+1)$. The winner's expected profi t for the $(y-1)$ th auction depends on this probability.

Let $E P_{1}(j, m, n)$ denote the winner's expected profi $t$ for the $j$ th auction in the series of $m$ auctions with $n$ bidders for the fi rst one. Likewise, let $\alpha_{1}(j, m, n)$ denote a bidder's ex-ante expected profi $\mathrm{t}$ from winning any one auction in the series of auctions from the $j$ th (for $1 \leq j \leq m$ ) to the $m$ th one. This profi t is:

$$
\alpha_{1}(j, m, n)=\sum_{y=j}^{m} \beta_{1}(y, j, m, n) E P_{1}(y, m, n)=\frac{1}{n-j+1} \sum_{y=j}^{m} E P_{1}(y, m, n)
$$

A defi nition for $E P_{1}(y, m, n)$ will be given in Theorem 1. Note that since there are $m$ objects, $\alpha(m+1, m, n)=0$.

Given that the number of objects is $m$ and the number of bidders for the fi rst auction is $n$, for auction $j, E S_{1}(j, m, n)$ denotes the expected surplus (surplus is what gets split between the auctioneer and the winning bidder, and it is synonymous with efficiency), and $E R_{1}(j, m, n)$ the expected revenue. Finally, for $n$ bidders, $E\left(f_{j}^{n}\right)$ and $E\left(s_{j}^{n}\right)$ denote the expected fi rst and second order statistic for the distribution $V_{j}$, from which the bidders draw their valuations for auction $j$.

Theorem 1. If each auction in a series is conducted using the second price rules, then the equilibrium for auction $j(1 \leq j \leq m)$ is:

$$
B_{j}^{1}\left(v_{j}\right)=\max \left\{0, v_{j}-\alpha_{1}(j+1, m, n)\right\}
$$


Proof. In order to find the equilibrium strategies, we begin with the last auction and then reason backwards. Recall that a bidder comes to know his valuation $v_{j}$ just before auction $j$ begins (i.e., after the previous $j-1$ auctions are over).

Consider auction $m$. The number of bidders for this auction is $n-m+1$. Since this is the last auction, the bidding strategies for it are the same as those for a single object auction [13]. Hence we get the following:

$$
\begin{aligned}
& E P_{1}(m, m, n)=E\left(f_{m}^{n-m+1}\right)-E\left(s_{m}^{n-m+1}\right) \\
& E S_{1}(m, m, n)=E\left(f_{m}^{n-m+1}\right) \\
& E R_{1}(m, m, n)=E\left(s_{m}^{n-m+1}\right)
\end{aligned}
$$

Now consider auction $j(1 \leq j<m)$. Consider bidder 1 and suppose that $b^{*}=$ $\max _{i \neq 1} b_{j}$ is the highest competing bid. By bidding $x_{j}=v_{j}-\alpha(j+1, m, n)$, the bidder will win if $x_{j}>b^{*}$ and lose if $x_{j}<b^{*}$. Now suppose that he bids $z_{1}<x_{j}$. If $x_{j}>z_{1} \geq b^{*}$, then he still wins and his profit is still $x_{j}-b^{*}$. If $b^{*}>x_{j}>z_{1}$, he still loses. But, if $x_{j}>b^{*}>z_{1}$, then he loses whereas if he had bid $x_{j}$ he would have made a positive profit. Thus, bidding less than $x_{j}$ can never increase his profit, but in some cases it may actually decrease it. A similar argument shows that it is not profitable to bid more than $x_{j}$.

Note that, for auction $j, \alpha_{1}(j+1, m, n)$ is a bidder's expected ex-ante profit from winning a future auction and is therefore constant (i.e., it is the same for all the bidders). Now, this constant may be greater than $v_{j}$ or less than it. Let $C$ denote the condition $\alpha_{1}(j+1, m, n)<\min \left\{v_{j}\right\}$. We first analyze the case where $C$ is true and then the case where $C$ is false ${ }^{2}$.

$C$ True: For this case, $v_{j}-\alpha_{1}(j+1, m, n)$ is always positive, so the equilibrium bids are:

$$
B_{j}^{1}\left(v_{j}\right)=v_{j}-\alpha(j+1, m, n)
$$

Since the equilibrium bid for auction $j$ decreases by $\alpha_{1}(j+1, m, n)$ (relative to a single object auction), the auctioneer's revenue decreases by the same amount. But the surplus for an auction (which is the sum of the winner's profit and the auctioneer's revenue) remains the same as that for a single object auction. Hence, we get the following:

$$
\begin{aligned}
& E P_{1}(j, m, n)=E\left(f_{j}^{n-j+1}\right)-E\left(s_{j}^{n-j+1}\right)+\alpha_{1}(j+1, m, n) \\
& E S_{1}(j, m, n)=E\left(f_{j}^{n-j+1}\right) \\
& E R_{1}(j, m, n)=E\left(s_{j}^{n-j+1}\right)-\alpha_{1}(j+1, m, n)
\end{aligned}
$$

$C$ False: For this case, $v_{j}-\alpha_{1}(j+1, m, n)$ may be negative, so the equilibrium bids are:

$$
B_{j}^{1}\left(v_{j}\right)=\max \left\{0, v_{j}-\alpha_{1}(j+1, m, n)\right\}
$$

Here, the expected surplus, the expected revenue, and the winner's expected profit for auction $j$ depend on the relationship of $\alpha_{1}(j+1, m, n)$ with the valuations for

\footnotetext{
${ }^{2}$ As mentioned in the Introduction, this proof is a generalisation of [1] which obtained the equilibrium for a setting with just two objects and where $C$ is true.
} 
the $n$ bidders. Let the valuations of the $n$ bidders be $v_{n}>v_{n-1}>\cdots>v_{1}$. Then, there are 3 cases we need to consider depending on this relationship. These cases are as follows: Case 1: $\alpha_{1}(j+1, m, n)<v_{1}$, Case 2: $v_{1}<\alpha_{1}(j+1, m, n)<v_{2}$, Case 3: $v_{2}<\alpha_{1}(j+1, m, n)$. We now analyze each of these cases. In what follows, we let $n_{z}$ denote the number of bidders whose bid for auction $j$ is zero.

Consider Case 1. For this case, $n_{z}=n$. All the bidders bid zero, and so the object is allocated to a randomly chosen bidder. The winner pays nothing, so the winner's profit, the surplus, and the revenue are:

$$
\begin{aligned}
& E P_{1}(j, m, n)=E\left(V_{j} \mid f_{j}^{n-j+1}<\alpha_{1}(j+1, m, n)\right)=E_{0} \\
& E S_{1}(j, m, n)=E P_{1}(j, m, n)=E_{0} \\
& E R_{1}(j, m, n)=0
\end{aligned}
$$

Consider Case 2. Here $n_{z}=n-1$ and only one bidder makes a positive bid while the rest bid zero. Thus, the object is allocated to the bidder with a positive bid. The winner pays nothing because the second highest bid is zero, so the winner's profit, the surplus, and the revenue are:

$$
\begin{aligned}
& E P_{1}(j, m, n)=E\left(f_{j}^{n-j+1} \mid s_{j}^{n-j+1}<\alpha_{1}(j+1, m, n)<f_{j}^{n-j+1}\right)=E_{1} \\
& E S_{1}(j, m, n)=E P_{1}(j, m, n)=E_{1} \\
& E R_{1}(j, m, n)=0
\end{aligned}
$$

Consider Case 3. For this case, $n_{z} \leq n-2$. Here, the winner pays the second highest bid so the winner's profit, the surplus, and the revenue are:

$$
\begin{aligned}
& E P_{1}(j, m, n)=E\left(f_{j}^{n-j+1} \mid \alpha_{1}(j+1, m, n)<s_{j}^{n-j+1}\right)- \\
& E\left(s_{j}^{n-j+1} \mid \alpha_{1}(j+1, m, n)<s_{j}^{n-j+1}\right)+ \\
& \alpha_{1}(j+1, m, n)=E_{2} \\
& E S_{1}(j, m, n)=E\left(f_{j}^{n-j+1} \mid \alpha_{1}(j+1, m, n)<s_{j}^{n-j+1}\right)=E_{2, s} \\
& E R_{1}(j, m, n)=E\left(s_{j}^{n-j+1} \mid \alpha_{1}(j+1, m, n)<s_{j}^{n-j+1}\right)- \\
& \alpha_{1}(j+1, m, n)=E_{2, r}
\end{aligned}
$$

By combining these three cases, we get:

$$
\begin{aligned}
& E P_{1}(j, m, n)=P_{0} E_{0}+P_{1} E_{1}+P_{2} E_{2} \\
& E S_{1}(j, m, n)=P_{0} E_{0}+P_{1} E_{1}+P_{2} E_{2, s} \\
& E R_{1}(j, m, n)=P_{2} E_{2, r}
\end{aligned}
$$

where the probability $P_{0}=\left(V_{j}\left(\alpha_{1}(j+1, m, n)\right)\right)^{n-j+1}$, the probability $P_{1}=$ $(n-j+1)\left(V_{j}\left(\alpha_{1}(j+1, m, n)\right)\right)^{n-j}\left(1-V_{j}\left(\alpha_{1}(j+1, m, n)\right)\right.$, and the probability $P_{2}=1-P_{0}-P_{1}$. Thus, given $\alpha_{1}(j+1, m, n)$, we can find $E P_{1}(j, m, n)$. Hence given $\alpha_{1}(y, m, n)$ for $j+1 \leq y \leq m$, we can find $\alpha_{1}(j, m, n)$ using Equation 2. 
Between Case 1, Case 2, and Case 3, if we assume it is Case 3 (note that under this assumption, the equilibrium bids are as given in Equation 7; so $E P_{1}, E S_{1}$, and $E R_{1}$ are as given in Equations 8, 9, and 10 respectively) then the expressions for fi nding $E P_{1}$ are easier to deal with because we do not have conditional expectations. Moreover, this case is important because, in general, for a large number of bidders, it is quite likely that $P_{2}=1$. This is because as $n$ increases, $E\left(f_{j}^{n}\right)-E\left(s_{j}^{n}\right)$ decreases [3], so a bidder's profi t from future auctions decreases and $\alpha_{1}$ also decreases. Hence, in the following sections, we will work under the assumption that $P_{2}=1$. We leave the analysis for the other cases (viz., Case 1 and Case 2) as part of future work.

For Case 3, we know from Equations 2 and 8, that the time to fi nd $\alpha_{1}(j, m, n)$ for $j=1$ is $\mathcal{O}(m)$. Since $\alpha_{1}(j, m, n)$ is defi ned recursively, once we fi nd $\alpha(1, m, n)$ it means that we have already found $\alpha_{1}(j, m, n)$ for $1<j \leq m$. Hence for auction 1 , the time to compute the equilibrium bid given in Equation 7 is also $\mathcal{O}(m)$. But the time to compute the equilibrium bid for all subsequent auctions is $\mathcal{O}(1)$.

\section{Equilibrium bids for scenario $S_{2}$}

This setting is the same as $S_{1}$ except that the bidders are now additionally uncertain about the number of objects for sale. This uncertainty is modelled as follows. We let $P L_{j}$ denote the probability that auction $j$ is the last auction. The probabilities $P L_{j}$ $\left(1 \leq j \leq m\right.$ ) are common knowledge to the bidders. Also, $P L_{m}=1$, i.e., all the bidders know that there are no more than $m$ objects for sale. As before, a bidder's signal (drawn from the p.d.f. $V_{j}$ for auction $j$ ) is his private information.

As before, the equilibrium bids for an auction are obtained using backward reasoning. However, for this setting, a bidder's ex-ante probability of winning auction $y$ in the series from $j$ to $m$ (denoted $\beta_{2}(y, j, m, n)$ ) depends on the probability that a given auction is the last one. Thus, we first fi nd $\beta(y, j, m, n)$.

To begin, consider the case where $m=2$. For this case, $0 \leq P L_{1} \leq 1$ and $P L_{2}=1$. Since $P L_{2}=1$ (i.e., the second auction is known by all to be the last one), $\beta_{2}(1,1,2, n)=1 / n$, and $\beta_{2}(2,1,2, n)=(1-1 / n)\left(1-P L_{1}\right)(1 /(n-1))$. Here $(1-1 / n)$ is a bidder's ex-ante probability of losing auction 1 , and $(1 /(n-1))$ is his probability of winning auction 2 . In general, for $j \leq y \leq m, \beta_{2}(j, y, m, n)$ is:

$$
\begin{aligned}
\beta_{2}(y, j, m, n) & =\left(\prod_{k=j}^{y-1}\left(1-P L_{k}\right)(1-1 /(n-k+1))\right) \times\left(\frac{1}{n-y+1}\right) \\
& =\frac{1}{n-j+1} \prod_{k=j}^{y-1}\left(1-P L_{k}\right)
\end{aligned}
$$

Let $\alpha_{2}(j, m, n)$ be a bidder's ex-ante expected profi t from winning any one auction in the series from auction $j$ to auction $m$ where:

$$
\alpha_{2}(j, m, n)=\sum_{y=j}^{m} \beta_{2}(y, j, m, n) E P_{2}(y, m, n)
$$


Obviously, $\alpha_{2}(m+1, m, n)=0$. For this setting, we let $B^{2}(\cdot)$ denote the equilibrium bids, $E P_{2}$ the winner's profi t, $E S_{2}$ the surplus, and $E R_{2}$ the revenue. The following theorem characterises the equilibrium bids:

Theorem 2. The equilibrium for auction $j(1 \leq j \leq m)$ is:

$$
B_{j}^{2}\left(v_{j}\right)=v_{j}-\alpha_{2}(j+1, m, n)
$$

Proof. As per Theorem 1.

It follows from Section 3 that for this equilibrium, the outcome for $1 \leq j \leq m$ is:

$$
\begin{aligned}
& E P_{2}(j, m, n)=E\left(f_{j}^{n-j+1}\right)-E\left(s_{j}^{n-j+1}\right)+\alpha_{2}(j+1, m, n) \\
& E S_{2}(j, m, n)=E\left(f_{j}^{n-j+1}\right) \\
& E R_{2}(j, m, n)=E S_{2}(j, m, n)-E P_{2}(j, m, n)
\end{aligned}
$$

Consider the effect of $P L_{j}$ on the bidding behaviour for auction $j$. We know from Section 3, that the equilibrium bids for auction $j$ depend on $\alpha_{1}(j+1, m, n)$. As per Equation $2, \alpha_{1}(j+1, m, n)$ depends on $\beta_{1}(y, j, m, n)$ (where $\left.j \leq y \leq m\right)$. Also, since $0 \leq P L_{j} \leq 1, \beta_{2}(y, j, m, n) \leq \beta_{1}(y, j, m, n)$ (see Equations 1 and 12). Consequently, we get:

$$
\alpha_{2}(j, m, n) \leq \alpha_{1}(j, m, n)
$$

Hence, from Equations 8 and 15 , it follows that $E P_{2}(j, m, n) \leq E P_{1}(j, m, n)$. From Equations 9 and 16 we get $E S_{2}(j, m, n)=E S_{1}(j, m, n)$. Also, from Equations 10 and 17 it follows that $E R_{2}(j, m, n) \geq E R_{1}(j, m, n)$.

Finally, we look at the time to fi nd the equilibrium bids. We know from Equations 13 and 15 , that the time to fi nd $\alpha_{2}(1, m, n)$ is $\mathcal{O}(m)$. Hence, for the fi rst auction, the time to compute the equilibrium bid given in Equation 14 is also $\mathcal{O}(m)$. By simply using the values obtained while fi nding $\alpha_{2}(1, m, n)$, we get the time to get the equilibrium bid as $\mathcal{O}(1)$ for all subsequent auctions.

\section{Equilibrium bids for scenario $S_{3}$}

This setting is the same as $S_{1}$ except that new bidders are allowed to join a series of auctions just before an auction in the series commences. As before, once a bidder joins the series, he does not leave until he wins an object or else the series ends. Here, the bidders are uncertain about the other bidders' valuations and the number of participating bidders. The uncertainty regarding the others' valuations is modelled as in Section 3 . The uncertainty regarding the number of participating bidders is modelled as follows. We let $P N(j, i)$ denote the probability that auction $j$ has $i$ bidders. The probabilities $P N(j, i)(1 \leq j \leq m)$ and $(1 \leq i \leq n)$ are common knowledge to the bidders. Note that all the bidders know that there are no more than $n$ bidders in any of the $m$ auctions. Note also that the number of bidders for auction $j$ is at least one less than the number of bidders for auction $j-1$. Finally, as before, a bidder's valuation for an object is his private information. 
We know from [8], that for a single object second price auction, the equilibrium bids for the case with a known number of bidders are the same as those for the case of an uncertain number of bidders - i.e., in both cases the bidders bid truthfully. On the basis of this result, we derive the equilibrium bids for sequential second price auctions as follows.

Consider the last auction. Since the equilibrium bids for this auction for the case with a known number of bidders are the same as those for case with an unknown number of bidders [8], the winner's expected profi t, the expected surplus, and the expected revenue are obtained from Equations 4, 5, and 6:

$$
\begin{aligned}
& E P_{3}(m, m, n)=\sum_{i=1}^{n} P N(m, i)\left(E\left(f_{m}^{i}\right)-E\left(s_{m}^{i}\right)\right) \\
& E S_{3}(m, m, n)=\sum_{i=1}^{n} P N(m, i) E\left(f_{m}^{i}\right) \\
& E R_{3}(m, m, n)=E S_{3}(m, m, n)-E P_{3}(m, m, n)
\end{aligned}
$$

We now reason backwards to obtain the bids for the previous auctions. Before doing so, we introduce some notation. Let $N$ be an $m$ element vector where $N_{j}$ denotes the number of bidders in auction $j$. Also, let $\bar{\alpha}_{3}(j, m, N)$ denote a bidder's ex-ante expected profi t from winning any one auction in the series from auction $j$ to auction $m$ when the number of bidders in each of these auctions is as given in $N$. Let $\bar{\beta}_{3}(y, j, m, N)$ be a bidder's ex-ante probability of winning auction $y$ in the series of auctions from $j$ to $m$, if the number of bidders in each auction is as given in $N$. For the case where the number of bidders is not known for the individual auctions, we let $\alpha_{3}(j, m)$ denote a bidder's ex-ante expected profi t from winning an auction in the series from $j$ to $m$. Then we get the following equations:

$$
\begin{gathered}
\bar{\beta}_{3}(y, j, m, N)=\frac{1}{N_{y}} \times\left(\prod_{k=j}^{y-1}\left(1-\frac{1}{N_{k}}\right)\right) \\
\bar{\alpha}_{3}(j, m, N)=\sum_{y=j}^{m} \bar{\beta}_{3}(y, j, m, N) \overline{E P} P_{3}(y, m, N)
\end{gathered}
$$

where $\bar{\alpha}_{3}(m+1, m, N)=0$ and $\overline{E P} P_{3}(y, m, N)$ is:

$$
\overline{E P} P_{3}(y, m, N)=E\left(f_{y}^{N_{y}}\right)-E\left(s_{y}^{N_{y}}\right)+\bar{\alpha}_{3}(y+1, m, N)
$$

Since the number of bidders for each auction lies between 1 and $n$, it follows that $\alpha_{3}(m-1, m)$ is:

$$
\sum_{N_{m-1}=1}^{n} \sum_{N_{m}=1}^{n}\left(P N\left(m-1, N_{m-1}\right) \times P N\left(m, N_{m}\right) \times \bar{\beta}_{3}(m-1, m-1, m, N) \times \overline{E^{-}} P_{3}(m-1, m, N)\right)
$$


and, in general, $\alpha_{3}(j, m)$ is:

$$
\alpha_{3}(j, m)=\sum_{N_{j}=1}^{n} \ldots \sum_{N_{m}=1}^{n}\left(\left(\prod_{i=j}^{m} P N\left(i, N_{i}\right)\right) \times \bar{\beta}_{3}(i, j, m, N) \times \bar{E} P_{3}(i, m, N)\right)
$$

Thus, the equilibrium bids for auction $j$ are the same as those for $S_{1}$ except that $\alpha_{1}$ in Equation 7 is replaced with $\alpha_{3}$ :

$$
B_{j}^{3}\left(v_{j}\right)=v_{j}-\alpha_{3}(j+1, m)
$$

Thus, as in Section 3, we get the following outcome for this scenario:

$$
\begin{aligned}
\forall_{j=1}^{m} E P_{3}(j, m) & =\sum_{k=1}^{n} P N(j, k) \times\left(E\left(f_{j}^{k}\right)-E\left(s_{j}^{k}\right)+\alpha_{3}(j+1, m)\right) \\
\forall_{j=1}^{m} E S_{3}(j, m) & =\sum_{k=1}^{n} P N(j, k) \times E\left(f_{j}^{k}\right) \\
\forall_{j=1}^{m} E R_{3}(j, m) & =E S_{3}(j, m)-E R_{3}(j, m)
\end{aligned}
$$

We now fi nd the relation between $\alpha_{1}(j, m, n)$ and $\alpha_{3}(j, m)$. We know from [3], that for auction $j, E\left(f_{j}^{n}\right)-E\left(s_{j}^{n}\right)$ is decreasing in $n$. In order to compare $\alpha_{1}$ and $\alpha_{3}$, the number of bidders in auction 1 must be the same in both cases - i.e., $P N(1, n)=1$ (and for $k<n, P N(1, k)=0$ ). Then we get:

$$
\alpha_{1}(j, m, n) \geq \alpha_{3}(j, m)
$$

Intuitively, this is so because in scenario $S_{1}$ the number of bidders decreases from one auction to the next, but in $S_{3}$ the number of bidders may increase. And if the number of bidders increases, a bidder's profi $t$ is bound to decrease.

Finally, for this scenario, the number of bidders for an auction lies between 1 and $n$. Thus, the vector $N$ can take $n^{m}$ possible values. For a given $N$, the time to find $\bar{\alpha}_{3}(1, m, N)$ is $\mathcal{O}(m)$. Thus, the time to fi nd $\alpha_{3}(1, m)$ is $\mathcal{O}\left(m n^{m}\right)$ and so is the time to fi nd the equilibrium bid for auction 1 . As before, for all remaining auctions, the time taken is $\mathcal{O}(1)$.

\section{Equilibrium bids for scenario $S_{4}$}

This scenario is the same as $S_{3}$ except that the bidders are now additionally uncertain about the number of objects for sale. This uncertainty about the number of objects is modelled with $P L$ (as per Section 4). Let $\bar{\alpha}_{4}(j, m, N), \bar{\beta}_{4}(y, j, m, N)$, and $\alpha_{4}(j, m)$ be analogous to $\bar{\alpha}_{3}(j, m, N), \bar{\beta}_{3}(y, j, m, N)$, and $\alpha_{3}(j, m)$ respectively. From Sections 4 
and 5, it is straightforward to obtain the equilibrium bids for $S_{4}$ as follows:

$$
\begin{gathered}
E P_{4}(m, m, n)=\left(\prod_{k=1}^{m-1}\left(1-P L_{k}\right)\right) \times \sum_{i=1}^{n} P N(m, i)\left(E\left(f_{m}^{i}\right)-E\left(s_{m}^{i}\right)\right) \\
E S_{4}(m, m, n)=\left(\prod_{k=1}^{m-1}\left(1-P L_{k}\right)\right) \times \sum_{i=1}^{n} P N(m, i) E\left(f_{m}^{i}\right) \\
E R_{4}(m, m, n)=\left(\prod_{k=1}^{m-1}\left(1-P L_{k}\right)\right) \times \sum_{i=1}^{n} P N(m, i) E\left(s_{m}^{i}\right) \\
\bar{\beta}_{4}(y, j, m, N)=\left(\prod_{k=j}^{y-1}\left(1-P L_{k}\right)\right) \times \bar{\beta}_{3}(y, j, m, N) \\
\bar{\alpha}_{4}(j, m, N)=\sum_{y=j}^{m} \bar{\beta}_{4}(y, j, m, N) \overline{E P}(y, m, N) \\
\alpha_{4}(j, m)=\sum_{N_{j}=1}^{n} \sum_{N_{m}=1}^{n}\left(\left(\prod_{i=j}^{m} P N\left(i, N_{i}\right)\right) \times \bar{\beta}_{4}(i, j, m, N) \times \overline{E P} P_{4}(i, m, N)\right) \\
B_{j}^{4}\left(v_{j}\right)=v_{j}-\alpha_{4}(j+1, m)
\end{gathered}
$$

As in Section 5, we get the following outcome for this scenario:

$$
\begin{aligned}
\forall_{j=1}^{m} E P_{4}(j, m) & =\sum_{k=1}^{n} P N(j, k) \times\left(E\left(f_{j}^{k}\right)-E\left(s_{j}^{k}\right)+\alpha_{4}(j+1, m)\right) \\
\forall_{j=1}^{m} E S_{4}(j, m) & =\sum_{k=1}^{n} P N(j, k) \times E\left(f_{j}^{k}\right) \\
\forall_{j=1}^{m} E R_{4}(j, m) & =E S_{4}(j, m)-E R_{4}(j, m)
\end{aligned}
$$

Since $\bar{\beta}_{4}(y, j, m, N) \leq \bar{\beta}_{3}(y, j, m, N)$ (see Equation 21) and $\overline{E P} 4(y, m, N) \leq \overline{E P} P_{3}(y, m, N)$ (see Equation 22) it follows that:

$$
\alpha_{4}(j, m) \leq \alpha_{3}(j, m) .
$$

Hence $E P_{4}(j, m) \leq E P_{3}(j, m)$ and $E R_{4}(j, m) \geq E R_{3}(j, m)$. In order to compare $\alpha_{4}$ and $\alpha_{2}$, we need to take $P N(1, n)=1$. Then, it is straightforward to see that

$$
\alpha_{4}(j, m) \leq \alpha_{2}(j, m, n) .
$$




\begin{tabular}{|c|c|c|c|c|}
\hline $\begin{array}{c}\text { Information } \\
\text { setting (1) }\end{array}$ & $\begin{array}{c}\text { Cumulative surplus } \\
\text { or Efficiency (2) }\end{array}$ & $\begin{array}{c}\text { Winner's } \\
\text { profit (3) }\end{array}$ & $\begin{array}{c}\text { Revenue } \\
\text { (4) }\end{array}$ & $\begin{array}{c}\text { Time to } \\
\text { find } \alpha\end{array}$ \\
\hline$S_{1}$ & $E\left(f_{j}^{n-j+1}\right)$ & $\begin{array}{c}E\left(f_{j}^{n-j+1}\right)-E\left(s_{j}^{n-j+1}\right) \\
+\alpha_{1}(j+1, m, n)\end{array}$ & $(2)-(3)$ & $\mathcal{O}(m)$ \\
\hline$S_{2}$ & $E\left(f_{j}^{n-j+1}\right)$ & $\begin{array}{c}E\left(f_{j}^{n-j+1}\right)-E\left(s_{j}^{n-j+1}\right) \\
+\alpha_{2}(j+1, m, n)\end{array}$ & $(2)-(3)$ & $\mathcal{O}(m)$ \\
\hline$S_{3}$ & $E\left(f_{j}^{n-\jmath+1}\right)$ & $\begin{array}{c}E\left(f_{j}^{n-\jmath+1}\right)-E\left(s_{j}^{n-j+1}\right) \\
+\alpha_{3}(j+1, m, n)\end{array}$ & $(2)-(3)$ & $\mathcal{O}\left(m n^{m}\right)$ \\
\hline$S_{4}$ & $E\left(f_{j}^{n-j+1}\right)$ & $\begin{array}{c}E\left(f_{j}^{n-j+1}\right)-E\left(s_{j}^{n-j+1}\right) \\
+\alpha_{4}(j+1, m, n)\end{array}$ & $(2)-(3)$ & $\mathcal{O}\left(m n^{m}\right)$ \\
\hline
\end{tabular}

Table 1. A summary of key results for auction $j(1 \leq j \leq m)$.

So we get $E P_{4}(j, m) \leq E P_{2}(j, m, n)$ and $E R_{4}(j, m) \geq E R_{3}(j, m, n)$. Intuitively, this happens because in $S_{4}$ the number of bidders from one auction to the next may increase while in $S_{2}$ this number strictly decreases by one. So a bidder's profit for $S_{4}$ is higher than that for $S_{2}$.

Finally, as per Sections 4 and 5, we get the time to solve Equation 23 for auction 1 as $\mathcal{O}\left(m n^{m}\right)$ and for all the remaining auctions as $\mathcal{O}(1)$.

\section{Related Work}

Since Ortega-Reichert's [11] seminal work on sequential auctions, a considerable amount of research effort has focussed on the subject. This work can be broadly divided into two categories [7,6]: that which deals with homogeneous objects and that which deals with heterogeneous objects. The analysis of sequential auctions for homogeneous objects is very well developed for the special case where no bidder is interested in more than one unit. Work in this category deals primarily with the study of sale price dynamics and shows that even when identical objects are sold in a series, the sale price varies from auction to auction. For instance, Weber [14] showed that in sequential auctions of identical private value objects, the expected sale price is the same for each auction. For sequential auctions with affi liated signals, Milgrom and Weber [10] showed that the expected selling price has a tendency to drift upward in later auctions. Finally, Mc Afee and Vincent [9] considered two identical private value objects and using the second price sealed bid rules, they showed that prices increase in later auctions.

On the other hand, work in the category of sequential auctions for heterogeneous objects includes $[1,4,5]$ and mainly deals with the effect of the agenda on the auction outcome. For instance, Bernhardt and Scoones [1] studied the effect of the agenda on the auction revenue by considering two private value objects using the second price rules. In the same setting, Elmaghraby [4] studied the effect of the agenda on auction effi ciency. Finally, for the setting described in Section 3, Fatima et al [5] consider objects with interdependent valuations and analyse the effect of uncertainty about these valuations on the auction outcome. 
Our work differs from the above in that we analyse four different information settings, while earlier work on sequential auctions has focused only on uncertainty about the bidders' valuations for the objects ${ }^{3}$. By analysing a range of information settings, our work complements and extends earlier work on sequential auctions.

\section{Conclusions and future work}

This paper analyzes sequential auctions for four different incomplete information settings with different sources of uncertainty. For each setting, we obtain the equilibrium bidding strategies and the resulting outcomes for the second price sealed bid rules. We then studied how the different sources of uncertainty affect the computational and the economic properties of the equilibrium solutions.

On the basis of the results given in Table1, we infer the following key conclusions for each individual auction:

1. Sequential auctions are equally effi cient in all the four information settings - see Column 2 in Table 1.

2. Between all the scenarios, the winner's expected profi $t$ for $S_{1}$ is the highest - see Equations 18, 20, 24, and 25.

3. Between all the scenarios, the auctioneer's revenue for $S_{4}$ is the highest - see Equations 18, 20, 24, and 25.

4. Since the revenue for scenario $S_{2}$, is higher than that for $S_{1}$, it is in the auctioneer's interest not to reveal information regarding which auction is the last one. This leaves the bidders' uncertain about whether or not there will be any future auctions and forces them to bid higher in a given auction.

5. The time to compute the equilibrium bids for the three scenarios depends on the time to compute the functions $\alpha_{1}, \alpha_{2}, \alpha_{3}$ and $\alpha_{4}$ (see Equations 3, 14, 19, and 23). But we know from Sections 3 to 6 that $\alpha_{1}, \alpha_{2}, \alpha_{3}$, and $\alpha_{4}$ depend on the players' common knowledge and are independent of their private value signals. Hence they can be computed before the first auction starts and these precomputations can be used when the auctions are run. Using these pre-computed values, it takes constant time to compute the equilibrium bids for each individual auction in each of the four scenarios.

There are several interesting direction for future work. Our present work assumes that the auction agenda is common knowledge to the auctioneer and the bidders. However the agents may equally well be uncertain about the agenda (i.e., the order in which the objects are auctioned). Since the auction outcome strongly depends on the agenda - if we change the agenda, then the outcome changes - we need to consider scenarios with an uncertain agenda and then fi nd the equilibrium bidding strategies. Second, we found the equilibrium bids using the second-price sealed bid rules. The analysis needs to be extended to other auction rules such as English and first-price sealed bid rules. Third, we focused on those scenarios where at least two bidders make a non-zero bid in each auction. We need to extend our analysis to scenarios where this condition is false.

\footnotetext{
${ }^{3}$ Even for this specific setting, the equilibrium bids were obtained for a restricted case - see the proof for Theorem 1 for details. Here, we determine the equilibrium for the general case without imposing any restrictions.
} 


\section{References}

1. D. Bernhardt and D. Scoones. A note on sequential auctions. American Economic Review, 84(3):653-657, 1994.

2. P. Dasgupta and E. Maskin. Efficient auctions. Quarterly Journal of Economics, 115:341388,2000

3. H. David. Order Statistics. Wiley, New York, 1969.

4. W. Elmaghraby. The importance of ordering in sequential auctions. Management Science, 49(5):673-682, 2003.

5. S. S. Fatima, M. Wooldridge, and N. R. Jennings. Sequential auctions for objects with common and private values. In Fourth International Conference on Autonomous Agents and Multi-Agent Systems, pages 635-642, Utrecht, Netherlands, 2005.

6. P. Klemperer. A survey of auction theory. Journal of Economic Surveys, 13(3):227-286, 1999.

7. V. Krishna. Auction Theory. Academic Press, 2002.

8. D. Levin and E. Ozdenoren. Auctions with uncertain number of bidders. Journal of Economic Theory, 118:229-251, 2004.

9. R. P. McAfee and D. Vincent. The declining price anomaly. Journal of Economic Theory, 60:191-212, 1993.

10. P. Milgrom and R. J. Weber. A theory of auctions and competitive bidding II. In The Economic Theory of Auctions. Edward Elgar, Cheltenham, U.K, 2000.

11. A. Ortega-Reichert. Models of competitive bidding under uncertainty. Technical Report 8 , Stanford University, 1968.

12. T. Sandholm and S. Suri. BOB: Improved winner determination in combinatorial auctions and generalizations. Artifi cial Intelligence, 145:33-58, 2003.

13. W. Vickrey. Counterspeculation, auctions and competitive sealed tenders. Journal of Finance, 16:8-37, 1961.

14. R. J. Weber. Multiple-object auctions. In R. Engelbrecht-Wiggans, M. Shibik, and R. M. Stark, editors, Auctions, bidding, and contracting: Uses and theory, pages 165-191. New York University Press, 1983.

15. M. P. Wellman, W. E. Walsh, P. R. Wurman, and J. K. McKie-Mason. Auction protocols for decentralised scheduling. Games and Economic Behavior, 35:271-303, 2001. 